\title{
Methodical Approach to Evaluating the Performance of the Institutional System of Crisis Management
}

\author{
Sergej Vladimirovich Kiselev ${ }^{1}$, Anna Vladimirovna Aksianova ${ }^{1}$ \& Albina Khatypovna Shagieva ${ }^{2}$ \\ ${ }^{1}$ Kazan National Research Technological University, Russian Federation \\ ${ }^{2}$ Kazan State Academy of Veterinary Medicine named N.E. Bauman, Russian Federation \\ Correspondence: Sergej Vladimirovich Kiselev, Kazan National Research Technological University. Russian \\ Federation.
}

Received: December 12, $2014 \quad$ Accepted: March 7, $2015 \quad$ Online Published: May 22, 2015
doi:10.5539/ass.v11n14p27

\begin{abstract}
The article considers the methodological aspects of application of statistical and information modeling to assess the performance of the institutional crisis management systems; proposes institutional attributes necessary to evaluate the performance; contains a conceptual model for evaluating the quality of the institutional crisis management system; suggests methods of estimation of the performance, efficiency and sustainability indicators, and discusses the possible directions of its further application to explore the issues of institutionalization of crisis management systems.
\end{abstract}

Keywords: institutional system of crisis management, institutional attributes, conceptual model for evaluating the institutional system quality, indicators of effectiveness and efficiency, value of the system entropy, statistical analysis of the ratio and sustainability of correlations

\section{Introduction}

Research in the theory of artificial intelligence and information, expert systems, game theory, probability and statistical solutions, mathematical statistics, statistical recognition theory is very important for the formation of methodological basic principles at the development of methods for evaluating the performance of the institutional system of crisis management. Despite the in-depth research in the field of mathematical logic, statistical and information modeling of these studies, objective difficulties occur in the economy with regard to building algorithms and programming processes of recognition of institutional attributes of a crisis management system.

These difficulties are related primarily to the limited linear nature of formalization at building the models, as a model plausibly displays the logical connection of object attributes, their informational value, but is not capable of adequate representation of its semantic correlations.

The assumption is methodologically justified that within the framework of the processes of institutionalization everything happening within an enterprise is important for understanding how and under which laws and regulations the distribution of resources outside the enterprise takes place. This assumption extends the systemic economic content of the general institutional approach to understanding the processes of crisis management (Auzan, 2010). With this approach, the crisis directly determines crisis management as a system of activities of particular institutional forms characterized by institutional attributes and indicators that exist over time, as the temporary basis and criteria for decision-making change in the circumstances of the anti-crisis program implementation.

The methodological approaches to evaluating the effectiveness of institutional systems of crisis management proposed in this study are based on the principles of system analysis, information modeling, and statistical analysis of system performance.

\section{Methodology of the research}

2.1 The Main Theoretical Concepts Defining the Principles for Evaluating the Effectiveness of the Institutional System of Crisis Management

Initially, the possibility of applying certain provisions of the information theory to the valuation of the results of institutionalization in crisis management was considered (Sukharev, 2005; Kirdina, 2007). We took the 
syntactical information measure as an information quantity measure. Other information measures were applied at evaluating the value of its institutional attributes (Polanyi, 1944)

The information gathered was processed using statistical methods: grouping, index and graphic plotting of time series. The results of processing were subjected to further analysis, which allowed describing the cause-and-effect relationships, on which the model of a particular crisis is based.

To construct such a predictive model, we used mainly three groups of forecasting methods complementing each other:

- Questioning, which reveals the opinion of the population or experts. This method is used in cases where the cause-and-effect relationship cannot be described analytically, i.e. using equations. Such methods, in particular, include the Delphi method, which is a series of sequential procedures for preparation and justification of a forecast, and the method of "brainstorming", when the decision is the product of collective work of experts in the course of a meeting held according to certain rules;

- Extrapolation, i.e. expectation of continuation of the future trends of processes represented in the form of time series of their indicators based on developed models of the regressive type. Extrapolation methods used are in the cases when information about the past is available in sufficient quantity and is based on the hypothesis of retention of previous tendencies;

- Analytical modeling, i.e. building a model that reflects the internal and external correlation of market factors. It is used when information about the past is scarce. However, there are some hypothetical ideas that allow developing a model and, on this basis, assessing the depth and duration of crisis phenomena (Information resources of the region: the principles of formation, organization, and specific structure, 2000)

Let us assume that there is a system of forecast states of the institutional system of crisis management, which includes $n$ states. Then, according to the results of information processing, it is possible to determine the statistical probability of occurrence of this state of effectiveness of the institutional system $R_{i}$.

Accordingly, the uncertainty of the system of possible outcomes of operation of the institutional system will be determined by the entropy value:

$$
U(R)=-\sum_{i} p\left(R_{i}\right) \log _{2} p\left(R_{i}\right)
$$

where:

$U(R)$ is theentropy of the institutional system performance;

$p\left(R_{i}\right)$ is the a priori probability of the performance of a particular institution of the system $R_{i}$;

As is known, institutional systems that combine equally probable outcomes of their activity have the maximum entropy. The entropy of such an institutional system consisting of $n$ equally probable states can be defined as:

$$
U(R)=-\sum_{i} p\left(R_{i}\right) \log _{2} p\left(R_{i}\right)=\sum \frac{1}{n} \cdot \log _{2} \frac{1}{n}=\log _{2} n
$$

For example, in the case of equally probable outcomes of operation of the institutional systems in the circumstances of a crisis and typology of economic crises, the value $n=20$ (Information resources of the region: the principles of formation, the organization, and specific structure, 2000). The value of entropy of such an institutional system will be equal to:

$$
U(R)=\log _{2} n=\log _{2} 20=4.34
$$

The entropy of the institutional system of crisis management will decrease in the case of entry of a certain amount of new or additional information to it, of both internal and external nature. At that, the amount of incoming information is comparable to the difference between the entropy of the institutional system before and after its receipt and can be expressed as follows (Kirdina, 2007):

$$
I R_{i}\left(k_{i}\right)=U\left(R_{i}\right)-U\left(R_{i} / k_{i}\right)
$$

where $I R_{i}\left(k_{i}\right)$ is the amount of information introduced into the system of the performance of the institutional system $R$ by the institutional attribute $k_{i}$;

$U\left(R_{i}\right)$ is the uncertainty of the performance of the institutional system prior to the entry of the information (initial entropy);

$U\left(R_{i} / k_{i}\right)$ is the uncertainty of the performance of the institutional system after receipt of the information by the attribute $k_{i}$. 
Thus, elimination of uncertainty leads to more effective control action of the institutional system of crisis management.

The value of the contributed information $I R_{i}\left(k_{i}\right)$ can be considered the information value of a specific institutional attribute $k_{i}$ with respect to the performance of this institution of the institutional system $R_{i}$, which reduces the entropy of the institutional system of crisis management as a whole.

The functional weight of a certain institutional attribute can be either a positive or a negative value, i.e. either reduce or increase the effectiveness of the institution and the entire institutional system of crisis management. The results of operation of one institution of crisis management, which has the highest institutional value, may be of significant importance and have high value for another institution of the system.

In accordance with the above, the total institutional value of the system by the institutional attribute can be estimated by the amount of information received as a result of monitoring the economy (Information resources of the region: the principles of formation, the organization and specific structure, 2000) The institutional weight of a simple attribute for the entire group of attributes of the institutional system is equal to:

$$
I R_{i}\left(k_{i}\right)=\sum_{i} p\left(R_{i}\right) I R\left(k_{i}\right)
$$

Using this relation, we can determine the optimal choice of the number of bits of an attribute. If the number of bits is increased, the institutional value of an attribute increases, and at the same time, the sampling size grows. In other words, the amount of information is almost predetermined, and we have only to build an optimal institutional (organizational) process of its accumulation.

Analyzing the opinions of experts in the field of adaptive effectiveness of institutions (Istomin, 2011; Sukharev, 2005; 2002; 2008; Petrosyan, 2007), we have proposed the following institutional attributes of the system (Table 1), which may be adopted for evaluation of their institutional value. We need to take into account the fact that the institutional value of a certain attribute depends on its previous value, or the process dynamics.

Table 1. Institutional attributes (indicators) of the system used at evaluating their institutional value

\begin{tabular}{l} 
Name of the institutional attribute \\
\hline Index - the ratio of completed arbitration cases in a given period (month, quarter, year) to the number of all \\
arbitration cases pending during the same period \\
Index - the ratio of restructured enterprises to bankrupt enterprises during the given period \\
Index - the ratio of job losses as a result of bankruptcy to the created (preserved) jobs as a result of restructuring \\
enterprises during the given period \\
Index - the ratio of the volume of income (loss) resulting from restructuring (bankruptcy) of enterprises to total \\
costs for maintaining the institutions of crisis management during the given period \\
Index - the rate of change (growth, reduction) of transaction costs for maintenance of crisis management institutions \\
Index - the growth rate of financial resources allocated for the maintenance of a certain institution of the crisis \\
management system for a certain period \\
Index -the ratio of the change (increase, reduction) in tax revenues to budgets of all levels as a result of restructuring \\
and financial recovery of economic entities to the value of the costs of the same budgets for maintenance of the \\
crisis management institutions \\
Index - the rate of change (growth, reduction) in transformation costs for the maintenance of crisis management \\
institutions for a certain period \\
Index -the ratio of newly established enterprises to the enterprises in bankruptcy (liquidation) for a certain period \\
Index - the growth rate of the balance of primary incomes of newly established business entities \\
$I_{g}$ \\
Index -the growth rate of the balance of primary incomes of economic entities who have undergone restructuring \\
Index - the proportion of vertically subordinate structures in the total number of structures (institutions) of the crisis \\
management system \\
Index - the proportion of horizontal structures in the total number of structures (institutions) of the crisis \\
management system \\
Index - the change in the dynamics of labor input in the implementation of crisis management programs
\end{tabular}

Using the concept of conditional probability, the information weight of availability of the Yth interval of the institutional attribute $k_{2}$ for the performance of a particular institution $R_{i}$ if the $j t h$ interval of the attribute is present $k_{1}$, can be defined as: 


$$
I_{i}\left(k_{2 Y} / k_{1 Y}\right)=\log _{2} \frac{p\left(k_{2 Y} / R_{i} k_{1 Y}\right)}{p\left(k_{2 Y} / k_{1 Y}\right)}
$$

Accordingly, provided the bit $j$ of the attribute is present, $k_{1}$ taking into account all the possible outcomes of the institutional system as a whole for the attribute $k_{2}$, the information value of the attribute $k_{2}$ will be equal to:

$$
I R_{i}\left(k_{2 j} / k_{1 j}\right)=-\sum p\left(k_{2 j} / R_{j} k_{1 j}\right) \cdot \log _{2} \frac{p\left(k_{2 Y} / R_{i} k_{1 Y}\right)}{p\left(k_{2 Y} / k_{1 Y}\right)}
$$

where: $I R_{i}\left(k_{2 j} / k_{1 j}\right)$ - quantitative expression of the information value of the attribute $k_{2}$.

In assessing the effectiveness of institutional systems, it is necessary to compare their institutional values (performance) and the amount of costs for each institutional attribute $k_{1}$. In this case, the cost value $C_{j}$ is understood as a set of all types of costs for the provision of information of a particular institution of the crisis management system (Balatsky, 2007; Kiselev \& Shagieva, 2011).

In accordance with this, we should take the following coefficient of efficiency by the attribute as a criterion for evaluating the effectiveness of the institutional system $k_{1}$ :

$$
\beta=\frac{\operatorname{IR}\left(k_{1}\right)}{\sum C_{j}}
$$

where:

$I R\left(k_{i}\right)$ - the information value of the institutional attribute;

$\sum C_{j}$ - the sum of the coefficients of the costs for providing a specific institution of the crisis management

system with information.

It is obvious that the coefficient of efficiency will have the maximum value if the amount of information $I R\left(k_{i}\right)$ is obtained at the lowest costs for its receipt.

Then, it becomes possible to compare the level of costs for collecting, archiving, processing, transmitting information through information and communication technologies in the system of crisis management institutions with the level of its effectiveness (see Figure 1).

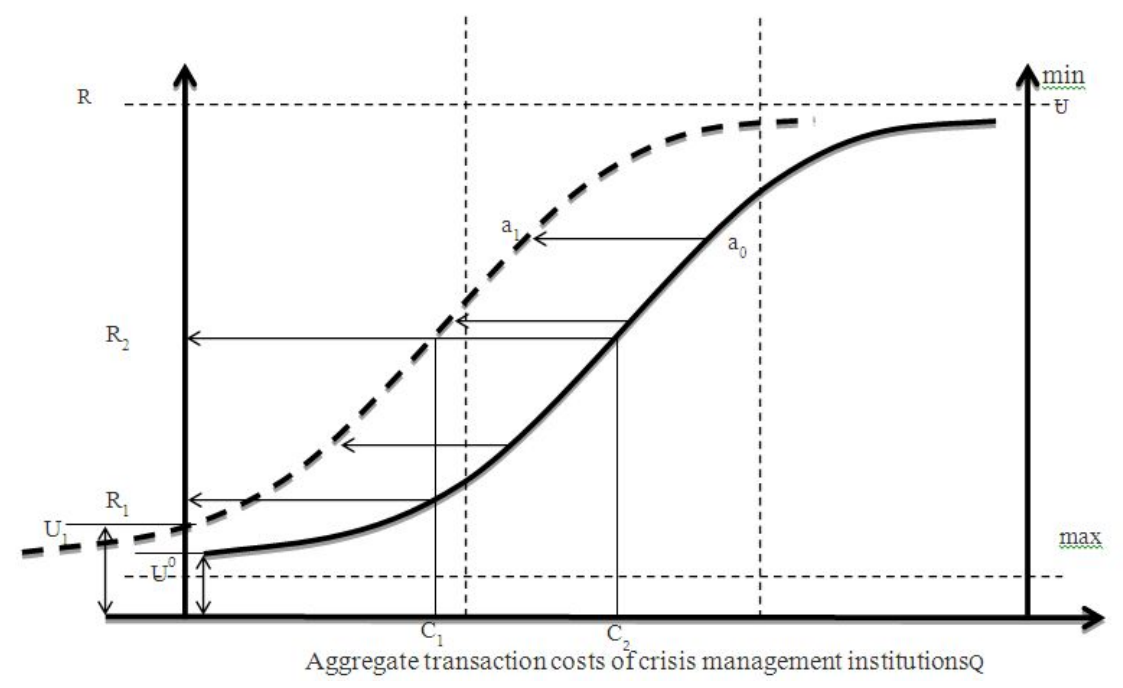

Figure 1. Dependence of the costs, the entropy, and the effectiveness of an institutional system of crisis management

On the left vertical of the proposed plot, the performance indicator of the institutional crisis management system $(\mathrm{R})$ is shown over time. The right vertical represents the value of entropy of the institutional crisis management system (U).

Thus, with increasing awareness of the institutional crisis management system and the decreasing level of its entropy, the costs for its acquisition grow, and simultaneously the overall efficiency of the system improves. However, at a certain point of achievement of a certain limit of the growth of costs for obtaining information and 
the minimum value of entropy, the effectiveness of the crisis management system ceases to improve and remains in a stable position. All this indicates the existence of marginal costs for reduction of the system entropy, when the growth of costs for achieving it is not justified by the absence of the dynamic improvement of the performance of individual institutions or the entire institutional system of crisis management.

\subsection{Specification of the Stages of the Effectiveness Evaluation of the Institutional System of Crisis Management}

The developed technique of evaluating the effectiveness of the institutional crisis management system involves the following sequence of stages of its implementation:

- identification of an object's belonging to a particular class or group of objects;

- identification of differences of the diagnosed object from the objects of its class by comparing its actual specifications to the core indicators;

- determination of permissible deviations from the core indicators;

- development of a technique of formation of institutional norms as the core indicators.

The generalization of all previously considered theoretical propositions was the decision to obtain statistical estimates of the quality of the institutional system of crisis management as a total of its following three components - effectiveness, efficiency, and sustainability (Kiselev \& Shagieva 2014; Kume, 2006). The conceptual statistic model of assessment of the quality of the institutional crisis management system represented in Table 2 was developed in accordance with the existing logic of effective business process management (Skripko, 2010).

Implementation of this approach requires additional specification of institutional attributes, shown in Table 1, in terms of their conditional division into the performance and resource attributes of the institutional system of crisis management. Accordingly, the selected performance attributes were actually taken as the targets of the system operation (Table 3).

Table 2. The conceptual statistical model of quality evaluation of the institutional crisis management system

\begin{tabular}{lll}
\hline Property & \multicolumn{1}{c}{ Definition } & \multicolumn{1}{c}{ The model of evaluation } \\
\hline 1. Performance & $\begin{array}{l}\text { The degree of implementation of the } \\
\text { planned activities and achievement of the } \\
\text { planned results } \\
\text { The ratio between the result achieved and } \\
\text { 2. Effectiveness }\end{array}$ & $\begin{array}{l}\text { The equity ratio of the achieved values of resource } \\
\text { the resources used }\end{array}$ \\
3. Stability & $\begin{array}{l}\text { Retaining the ability to achieve a result } \\
\text { within the established goals and resources }\end{array}$ & $\begin{array}{l}\text { Equity ratio of the reached values of the development } \\
\text { indicators of the system and its maximum values }\end{array}$ \\
\hline
\end{tabular}

Table 3. The matrix of indicators of the institutional crisis management system

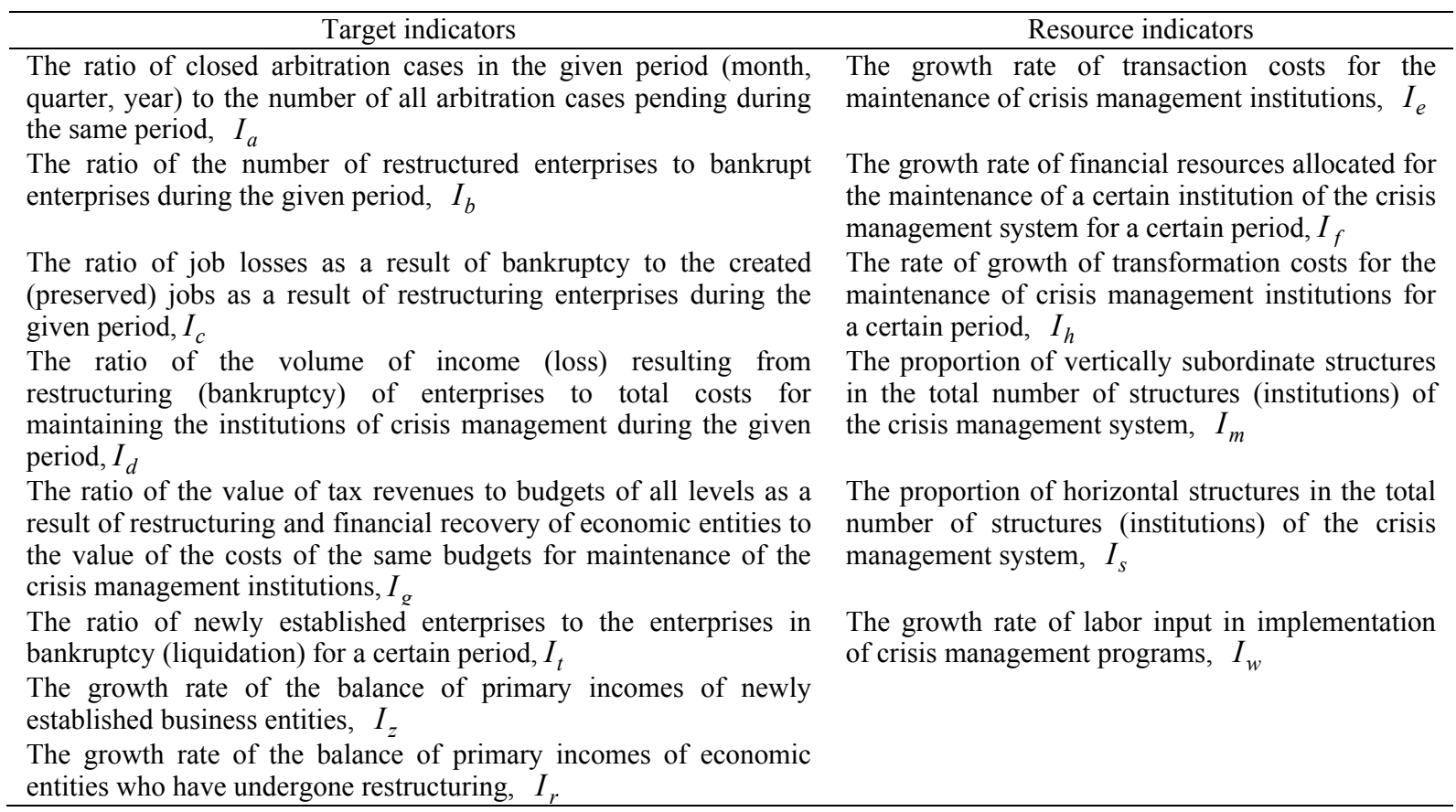


In accordance with the previously determined conceptual statistical model, we initially were to determine the effectiveness of the system by the ratio of the actual targets with the planned baselines:

$$
\begin{gathered}
I_{i}^{R}=\frac{I_{i}^{\text {actual }}}{I_{i}^{\text {basic }}} \\
\text { or } I_{a}^{R}=\frac{I_{a}^{\text {actual }}}{I_{a}^{\text {basic }}}, I_{b}^{R}=\frac{I_{b}^{\text {actual }}}{I_{b}^{\text {basic }}}, I_{c}^{R}=\frac{I_{c}^{\text {actual }}}{I_{c}^{\text {basic }}} \text { etc. }
\end{gathered}
$$

The overall performance indicator of the system was represented as the geometric mean of the obtained values $I_{i}^{R}$ :

$$
I_{R E S}=\sqrt[n]{\prod_{i} I_{i}^{R}}
$$

where $n$ is the number of targets (in this case $n=8$ ).

The second component (efficiency) was expressed through the value of the difference between the standard deviations of the performance values of the resource and target attributes (by the absolute value):

$$
I_{E F F}=\frac{1}{K} \sum_{k}\left(1-\left|\sigma_{R E S}-\sigma_{k}\right|\right),
$$

where $\mathrm{k}=1$ to $\mathrm{K}-\mathrm{it}$ is the number of consolidated groups of resource attributes of the system.

In this case, three consolidated groups were separated from the six institutional resource attributes: the financial component (the indicators $-I_{e}, I_{f}$ and $I_{h}$ ), the organization and structure component (the indicators $I_{m}$ and $I_{s}$ ), and the labor input $-I_{w}$.

The consolidated groups of resource indicators were singled out in order to identify the imbalance between the resulting states of the system and its input factors in terms of statistical evaluation of variation (scatter) (Balatsky, 2007). This statement does not exclude the possibility in future to expand the range of resource indicators by combining them in other groups of indicators characterizing the initial state of the system.

In this case, estimates of the variation in each of the three consolidated groups of institutional resource attributes were obtained:

$$
\sigma_{f i n}=\sqrt{\frac{1}{3}\left(\left(I_{e}^{R}-1\right)^{2}+\left(I_{f}^{R}-1\right)^{2}+\left(I_{h}^{R}-1\right)^{2}\right)},
$$

where $I_{e}^{R}=\frac{I_{e}^{\text {actual }}}{I_{e}^{\text {basic }}}, I_{f}^{R}=\frac{I_{f}^{\text {actual }}}{I_{f}^{\text {basic }}}, I_{h}^{R}=\frac{I_{h}^{\text {actual }}}{I_{h}^{\text {basic }}}$

$$
\sigma_{s t r}=\sqrt{\frac{1}{2}\left(\left(I_{m}^{R}-1\right)^{2}+\left(I_{s}^{R}-1\right)^{2}\right)},
$$

where $I_{m}^{R}=\frac{I_{m}^{\text {actual }}}{I_{m}^{\text {basic }}}, I_{s}^{R}=\frac{I_{s}^{\text {actual }}}{I_{s}^{\text {basic }}}$

$$
\sigma_{w}=\sqrt{\left(I_{W}^{R}-1\right)^{2}} \text { where } I_{w}^{R}=\frac{I_{w}^{\text {actual }}}{I_{w}^{\text {basic }}} .
$$

As a result, the overall assessment of the effectiveness of the institutional system of crisis management was expressed as:

$$
I_{E F F}=\frac{1}{3} \sum_{k}\left(1-\left|\sigma_{R E S}-\sigma_{k}\right|\right)
$$

where $\sigma_{R E S}$ is the standard deviation value in terms of effectiveness and $\sigma_{R E S}=\sqrt{\frac{1}{n}\left(\sum_{i}\left(I_{i}^{R}-1\right)^{2}\right)}$. 
The stability property, which is the third component of the proposed conceptual statistic model of assessment of the institutional system operation quality, was suggested to express through the considered earlier characteristic of the entropy of its performance (Maslova \& Mekhantseva, 2010; Aleksandrovskaya, 2014). As the maximum entropy will correspond to an institutional system, in which all the observed performance values will be distributed evenly, i.e. have equal probability values, the stability property was assessed through the entropy of the system by the observed values of the nearly maximum performance:

$$
I_{E N T R}=\left(1-\frac{U(p)}{U_{\max }(p)}\right) * 100
$$

where:

$U(p)=-\sum_{i=1}^{n} p_{i} \log _{2} p_{i}$ is the entropy for all of the obtained values of the system performance $\left(I_{i}^{R}\right) p_{i}=\frac{f_{i}}{\sum f_{i}}$ is the probability of a performance value within the range of the scale of the system performance values, $f_{i}$ is the frequency of occurrence of the performance indicator values,

$$
\sum_{i} f_{i}=n \quad U_{\max }(p)=-\sum_{i=1}^{n} p_{i} \log _{2} p_{i}, \quad p_{1}=p_{2}=\ldots .=p_{n}=\frac{1}{n} .
$$

Value $I_{\text {ENTR }}$ makes it possible to evaluate the ability of the institutional system in the observed state to achieve planned results in all performance indicators. The higher value $I_{E N T R}$, the less stable the observed system is. A state of complete stability (a hypothetical case) will match zero value of $I_{\text {ENTR }}$.

In terms of practical usage, the suggested model should provide opportunities for studying the three components of the model over time.

At that, we decided to provide calculation of the first component - the performance - under the same principle, namely, as the geometric mean of all the performance indicators of the system with the only difference that the values calculation requires providing totals for the series of periods.

When calculating the second component, we can proceed to the concept of evaluating the effectiveness of relations between the target and resource indicators of the institutional system of crisis management:

$$
\mathrm{I}_{\mathrm{EFF}}=\sqrt\left[\llcorner]{\prod_{1} \eta_{1}},\right.
$$

where $\mathrm{l}=1$ to $\mathrm{L}-$ it is the number of significant empirical correlation ratios $\eta_{l}$ obtained by analysis of variance of dependencies between the observed performance indicators of the institutional system $\left(I_{i}^{R}\right)$ and the performance characteristics of the resource attributes of the system (in this case - $I_{e}^{R}, I_{f}^{R}, I_{h}^{R}, I_{m}^{R}, I_{s}^{R}$, and $\left.I_{w}^{R}\right)$.

According to the standard statistical procedure, under the rule of addition of variances, the empirical correlation ratio is defined as the ratio of between-group variance (characterizing variability of the selected performance indicator of the system $\left(I_{i}^{R}\right)$ under the influence of the chosen performance indicator of the resource attribute) to the total variance (characterizing the variability $I_{i}^{R}$ under the influence of various factors):

$$
\eta=\sqrt{\frac{\sigma_{y x}^{2}}{\sigma^{2}}}, \quad \sigma^{2}=\sigma_{y x}^{2}+\overline{\sigma^{2}},
$$

where $\sigma^{2}$ is the total variance, $\sigma_{y x}^{2}$ is the intergroup variance, $\overline{\sigma^{2}}$ is the medium of the intra-group variances (it characterizes the variance of values of the measure $I_{i}^{R}$ under the influence of random factors).

Such an analysis requires building analytical groups, in which the all-time values of the selected indicator for the resource attribute performance are divided into groups at certain intervals, and for each resulting group the average value of the observed indicator of the system performance is calculated $\left(I_{i}^{R}\right)$. Thus, the set of all possible combinations of the system performance attributes and effectiveness of the resource attributes are set.

Traditionally, at the practical interpretation of the values of empirical correlation ratio, the neighboring relationship is estimated by the degree of approximation of the value $\eta$ to one. The closer the obtained value $\eta$ to one is, the stronger the correlation ratio between the analyzed attributes is. However, the statistical significance of the importance of the correlation by the value $\eta$ with account of the sampling size is determined 
by Fisher's statistical test, according to which the neighboring relationship is substantial and statistically significant if the ratio of the estimated value of the Fisher criterion is greater than the critical (table) value at the set 5\% level of significance (Haken, 1991; Stengers \& Prigozhin, 1986).

As is well known, the calculated value by the Fisher test is defined as the ratio of intergroup and intragroup variances estimates: $F_{\text {estimated }}=\frac{S_{1}^{2}}{S_{2}^{2}}$.

Thus, despite the fact that the final performance indicator $I_{E F F}$ accounts only significant correlations, and, consequently, the system efficiency will be higher if the value $I_{E F F}$ is closer to one, the identification of minor correlations will contribute to taking appropriate corrective management decisions to improve the efficiency of using the resource components of the institutional system.

The third property of the considered system was suggested to be regarded over time as stable connection. In this case, the calculation technique using entropy values actually remains the same, but the values of entropy should be calculated with account of the communications correlations significant by their closeness:

$$
I_{E N T R}=\left(1-\frac{U(p)}{U_{\max }(p)}\right) * 100,
$$

where $U(p)=-\sum_{i=1}^{n} p_{i} \log _{2} p_{i}$ is the entropy of all the obtained values of significant correlations of the performance indicators of the system $I_{i}^{R}$ with relevant performance indicators of resources $-I_{e}^{R}, I_{f}^{R}, I_{h}^{R}, I_{m}^{R}, I_{s}^{R}$, and $I_{w}^{R} ; p_{i}=\frac{f_{i}}{\sum f_{i}}$ is the probability of occurrence of the value $\eta$ in the respective interval of their scale (for example, on a Chaddock scale), $f i$ is the frequency of occurrence of the value $\eta$ in the respective interval of their scale; $\sum_{i} f_{i}=n^{*} M, M$ is the number of resource indicators of the system, in this case $M=6$ ).

$U_{\max }(p)=-\sum_{i=1}^{n} p_{i} \log _{2} p_{i}, p_{1}=p_{2}=\ldots=p_{n}=\frac{1}{n * M}$.

\section{Results}

The institutional system assumes varying the alternative projects for the company to overcome the crisis or prevent it, which requires appropriate information support that would objectively reflect the real processes in the system. Therefore, the institutional system should be treated as an institution of stable correlation between their functional content and its verified performance. Consequently, the institutional system of crisis management has a definite logic of the operation expressed in the following sequence of actions:

- if timely (A);

- reliably and with sufficient completeness of the information (B);

- the substantiated technique of its processing $(\mathrm{C})$;

- anti-crisis measures are implemented (D);

- with a high degree of probability, the achievement of the assigned goal can be guaranteed, as a result of the effective operation of the institutional system of crisis management.

The implementation of a systematic approach, statistical modeling, and analysis of the correlations of the key parameters of the system is an essential condition of identifying the synergistic effects in the interaction of elements of the institutional system of crisis management (Minashkin, 2012; Shuman \& Bulmer, 2004). With this approach, it is possible not only to establish what the outcome of the system is and how well it is achieved, but also to predict achievement of the result and its effectiveness in the next period through the sustainability of correlations in the system. 
On the other hand, identification of significant correlation ratios will facilitate priority allocation of management efforts in regulation of exactly those correlations, which have potential synergistic effect.

Analysis of stability of the correlations can be the basis for the development of strategies of the institutional system development. The presence of stable correlations indicates the possibility of adjusting the strategy, since there is a change in the responsiveness of target states of the system depending on a particular resource component.

\section{Discussion}

The proposed approach to the estimation of the development quality of the institutional system of crisis management in the concept of unity of the three components - efficiency, effectiveness, and sustainability - and in view of its development over time with further transition to the analysis of the key significant correlations in the system can be implemented both globally and regionally. In the latter case, the analysis of correlations becomes even more valuable in terms of accounting the institutional singularities and the institutional originality of the regional systems at the development of adequate strategic decisions and programs for the development of meso-level institutional systems of crisis management.

It should be noted that the specification of the proposed institutional attributes of the system of crisis management could be adjusted to reflect the dynamics of institutional transformations, as the cyclical development of the institutional system of crisis management is accompanied by emergence of its new, specific qualities.

However, the implementation of the proposed approach will be possible only in dealing with the organization of statistical accounting of the aggregate institutional attributes at the relevant levels (country, region). To obtain reliable statistical estimates for the proposed model, one should have a database of dynamic rows for the suggested institutional attributes (at least for 5 years).

\section{Conclusion}

Thus, the identification and study of specific properties of information and its role in the process of institutionalization of crisis management, and techniques for assessing the effectiveness, efficiency, and sustainability of the institutional system of crisis management allow us to formulate certain conclusions:

- the continuous process of bankruptcy, liquidation, restructuring, and simultaneous emergence of new enterprises evidences a constant process of renewal and growth of information flows, which significantly raises the significance of the information value of the institutional attributes of the structural institutions of the institutional system of crisis management as a whole;

- the high entropy of the market macro environment of enterprises significantly reduces the manageability, the level of information monitoring of the changes in the situation development, and the probability of forecasting;

- the constant threat of crisis conditions determines the selection and amplification of prognostic, anti-crisis, warning functions of crisis management institutions in relation to the stabilization of the economic development of enterprises.

In addition, one of the most promising directions in the information support of the institutional system of crisis management is the expert assessment of the financial position of enterprises and the bankruptcy procedures, the results of which are the information-analytical framework for adaptation of the institutions of the crisis management system.

Certain generalization of the institutional theory is required in order to synthesize the diversity of approaches to assessing the role of the institutional component in the development of the financial and economic state of an enterprise, its organizational structure, and management system. This is especially important when evaluating complex objects, when you need to rely on adaptive, learning and self-learning systems.

\section{References}

Alexandrovskaya, Yu. (2014). Statistical modeling of the quality of an industrial enterprise. Bulletin of the Kazan University of Technology, 17(15), 354-361.

Auzan, A. (2010). Institutional Economics (p. 254). Moscow: Infra-M.

Balatsky, E. (2007). Fuzzy institutions, culture of the population, and institutional entropy. Society and Economy, $5-6,37-51$.

Information resources of the region: the principles of formation, organization, and specific structure (p. 167). (2000). Novosibirsk: SPSTL SO RAS. 
Istomin, S. (2011). Analysis of the effectiveness of the institutional mechanism of Russia. Bulletin of the Chelyabinsk State University, Economy, 31(246), 65-70.

Khaken, G. (1991). Information and self-organization, the macroscopic approach to complex phenomena ( $\mathrm{p}$. 240). Moscow: Mir.

Kirdina, S. (2007). X-effectiveness and X-economics: synthesis of theoretical approaches. Moscow.

Kiselev, C., \& Shagieva, A. (2011). Adaptation of the European experience of bankruptcy procedures at enterprizes. Bulletin of the Kazan University of Technology, 10, 263-271.

Kiselev, C., \& Shagieva, A. (2014). Simulation of effectiveness evaluation of the institutional system of crisis management. International Journal of Applied and Basic Research, 9(3), 114-118.

Kume, H. (2006). Statistical methods for quality improvement: Study Guide. Moscow: Finance and Statistics.

Maslova, N., \& Mehantseva, K. (2010). Development of modern approaches to the study of "organization quality": the statistical aspect. Economics, Statistics, Computer Science. Bulletin of the EMA, 5, 104-111.

Minashkin, B. (Ed.). (2012). Methodology of statistical research on socio-economic processes: Scientific Edition (p. 387). Moscow: YUNITY-DANA

Petrosyan, D. (2007). Institutional pathology of the national economy. Auditing and Financial Analysis, 2.

Polanyi, K. (1944). Self-regulating Market and Fictitious Commodities: Labor, Land and Money. In the Great Transformation (Vol. 1, pp. 68-76). New York: Farrar \& Pinehart.

Shuman, H., \& Bulmer, M. (2004). The random probe: A technique for evaluating the validity of closed questions. In Questionnaires (Vol. 3). Thousand Oaks: SAGE Publications.

Skripko, L. (2010). Quality and competitiveness management (p. 127). St. Petersburg: UNECON Publishing House.

Stengers, I., \& Prigozhin, I. (1986). Order out of chaos. Moscow: Nauka.

Sukharev, O. (2002). Concepts of economic dysfunction and evolution of a company. Issues of Economics, 10.

Sukharev, O. (2005). The problem of quality of institutional development. Economic Theory, 2, 67-85.

Sukharev, O. (2008). Towards a new theory of economic efficiency. Investments in Russia, 12, 22.

\section{Copyrights}

Copyright for this article is retained by the author(s), with first publication rights granted to the journal.

This is an open-access article distributed under the terms and conditions of the Creative Commons Attribution license (http://creativecommons.org/licenses/by/3.0/). 